This is an Open Access article licensed under the terms of the Creative Commons AttributionNonCommercial-NoDerivs 3.0 License (www.karger.com/OA-license), applicable to the online version of the article only. Distribution for non-commercial purposes only.

\title{
Urinary Neutrophil Gelatinase-Associated Lipocalin Measured on Admission to the Intensive Care Unit Accurately Discriminates between Sustained and Transient Acute Kidney Injury in Adult Critically III Patients
}

\author{
Hilde R.H. de Geus ${ }^{c}$ Jessica G. Woo ${ }^{a} \quad$ Yu Wang ${ }^{\text {a }}$ Prasad Devarajan $^{\text {b }}$ \\ Michiel G. Betjes ${ }^{d}$ Jos L.M.L. le Noble ${ }^{c}$ Jan Bakker ${ }^{c}$ \\ ${ }^{a}$ Division of Biostatistics and Epidemiology, and ${ }^{b}$ Departments of Pediatrics, Developmental \\ Biology, Nephrology and Hypertension, Cincinnati Children's Hospital Medical Center, Cincinnati, \\ Ohio, USA; Departments of ${ }^{\mathrm{C} I n t e n s i v e ~ C a r e ~ a n d ~}{ }^{\mathrm{d}}$ Nephrology, Erasmus University Medical Center, \\ Rotterdam, The Netherlands
}

\section{Key Words}

Acute kidney injury $\cdot$ Cystatin C $\cdot$ Intensive care unit $\cdot$ Neutrophil gelatinase-associated lipocalin $\cdot$ Renal replacement therapy $\cdot$ Sustained acute kidney injury

\begin{abstract}
Background: First we aimed to evaluate the ability of neutrophil gelatinase-associated lipocalin (NGAL) and cystatin- $\mathrm{C}(\mathrm{CyC})$ in plasma and urine to discriminate between sustained, transient and absent acute kidney injury (AKI), and second to evaluate their predictive performance for sustained AKI in adult intensive care unit (ICU) patients. Methods: A prospective cohort study of 700 patients was studied. Sample collection was performed over 8 time points starting on admission. Results: After exclusion 510 patients remained for the analysis. All biomarkers showed significant differentiation between sustained and no AKI at all time points ( $p \leq 0.0002)$ except for urine $\mathrm{CyC}(\mathrm{uCyC})$ on admission $(p=0.06)$. Urine NGAL ( $\mathrm{UNGAL}$ ) was the only biomarker significantly differentiating sustained from transient AKI on ICU admission ( $p=0.02)$. Individually, UNGAL performed better than the other biomarkers (area under the curves, AUC = $0.80,95 \%$ confidence interval, $\mathrm{Cl}=0.72-0.88$ ) for the prediction of sustained AKI. The combination with plasma NGAL (pNGAL) showed a nonsignificant improvement ( $A U C=0.83,95 \% \mathrm{Cl}=$ 0.75-0.91). The combination of individual markers with a model of clinical characteristics (MDRD
\end{abstract}


eGFR, $\mathrm{HCO}_{3}{ }^{-}$and sepsis) did not improve its performance significantly. However, the integrated discrimination improvement showed significant improvement when UNGAL was added ( $p=$ 0.04). Conclusions: UNGAL measured on ICU admission differentiates patients with sustained AKI from transient or no-AKI patients. Combining biomarkers such as PNGAL, UNGAL and plas$\mathrm{ma} \mathrm{CyC}$ with clinical characteristics adds some value to the predictive model.

Copyright $\odot 2011$ S. Karger AG, Basel

\section{Introduction}

Acute kidney injury (AKI) is common in adult hospitalized patients and associated with significant morbidity and mortality (up to 50\%) [1-3]. AKI in critically ill patients is rarely an isolated event but usually more often part of a more multisystemic immunological condition contributing to remote organ dysfunction.

However, several large cohort studies have established a strong independent relationship between the severity of AKI and its associated mortality. Moreover, even small changes in kidney function and periods of transient azotemia have considerable impact on outcome [4]. In order to initiate timely therapies, it seems essential to discriminate patients with high risk for sustained AKI from patients with lower risk. Unfortunately, at the moment, specific therapies for AKI are lacking even though experimental animal data show promising possibilities. One major setback is that these new agents show promise only in the early reversible stages of AKI [5]. Therefore, it is essential to possess accurate tools for early detection of sustained AKI.

The rise in serum creatinine (SCr) levels is still used for the diagnosis of AKI; consequently it is the basis of AKI definitions such as the RIFLE (risk-injury-failure) criteria. However, it has a poor predictive value for AKI in the early stage of renal injury. In recent years, a number of novel biomarkers have been studied for their potential to detect AKI in its early stage [6].

Neutrophil gelatinase-associated lipocalin (NGAL) is a $25-\mathrm{kDa}$ protein combined with a siderophore, produced by epithelial tissues throughout the human body. It is involved in nephrogenesis, induction of nephritic structures from mesenchymal stroma cells, protection against ischemic tubular damage and in iron transportation [7-10]. Under normal conditions, plasma NGAL (pNGAL) is excreted via glomerular filtration and undergoes complete proximal tubular reabsorption. Urine NGAL (uNGAL) is expressed in the distal tubular segments where it is part of bacterial defense mechanisms.

Cystatin- $\mathrm{C}(\mathrm{CyC})$ is a $13-\mathrm{kDa}$ nonglycosylated cysteine protease inhibitor produced by all nucleated human cells at a constant rate unaffected by muscle mass. Plasma CyC (pCyC) is also excreted by glomerular filtration and has a similar catabolic pathway as pNGAL; therefore it is not normally found in urine in significant amounts. Some evidence suggests that $\mathrm{pCyC}$ is a superior functional biomarker compared to $\mathrm{SCr}$ especially to detect acute changes in estimated glomerular filtration rate (eGFR) $[11,12]$.

Several recent clinical studies have reported encouraging predictive properties of NGAL and CyC for AKI development [12-20]. Despite the reasonable individual biomarker performances, it is recognized that biomarker combinations or combined models with clinical parameters might provide additional accuracy to successfully guide clinical decision making [16].

The purpose of this study was to evaluate the discriminative properties of pNGAL, uNGAL, pCyC and uCyC for various AKI disease states and the performance of the biomarkers on the prediction of developing sustained AKI. 


\section{Materials and Methods}

\section{Study Population}

Between September 1, 2007, and April 1,2008, all consecutively admitted patients to the adult ICU of the Erasmus University Medical Center in Rotterdam, the Netherlands (where general surgical, trauma, medical, neurological and neurosurgical patients but not cardiac surgery patients are treated) were considered for participation $(n=700)$. Exclusion criteria were age $<18$ years, readmission during the study period (only the first admission records were included), refusal of informed consent, nephrectomy, chronic kidney disease (CKD, stages 3, 4 and 5 based on eGFR criteria calculated with the baseline SCr values) and renal transplantation. Written informed consent was obtained from all participants or their legal representative with a deferred consent policy [21]. The study was approved by the institutional review board.

\section{Study Protocol}

On ICU admission $(\mathrm{T}=0)$ and at 7 time points thereafter $(\mathrm{T}=4,8,24,36,48,60$ and $72 \mathrm{~h}$ ) plasma and urine sampling was performed using an intra-arterial line and a urine catheter for spot urine. The samples were immediately processed in the hospital's laboratory and the supernatants were stored at $-80^{\circ} \mathrm{C}$. NGAL and $\mathrm{CyC}$ concentrations were measured by Biosite Inc. in San Diego (Calif., USA) using the Triage ${ }^{\circledR}$ immunoassay. SCr values were measured in the hospital's clinical chemical laboratory (using the enzymatic kit produced by Roche which is traceable to a reference method based on isotope dilution-mass spectrometry) at the time of admission and thereafter daily at 6:00 a.m. eGFR was calculated using the Modification of Diet in Renal Disease (MDRD) Study Equation [22] and baseline SCr was defined as the patient's steady state level 4 weeks prior to ICU admission. If not available, the admission value was used as the baseline. AKI was defined according to the RIFLE classification [23]. The RIFLE classification is based on the rise in SCr compared to a baseline value. Risk represents a 1.5-2 times increase, injury a 2-3 times increase and failure a $>3$ times increase. Sustained AKI was defined as any AKI occurring and persisting for $>24 \mathrm{~h}$ after admission. Transient AKI was defined as AKI presenting only once and reaching normal SCr levels within $24 \mathrm{~h}$. Other variables recorded included age, gender, body mass index, bicarbonate levels $\left(\mathrm{HCO}_{3}{ }^{-}\right)$, blood urea nitrogen $(\mathrm{BUN})$ content, white blood cell count (WBC), APACHE II (Acute Physiology and Chronic Health Evaluation) score, SOFA (Sequential Organ Failure Assessment) score, cumulative urine output, initiation of renal replacement therapy, diagnosis at discharge, length of ICU stay, ICU mortality and hospital mortality.

\section{NGAL Measurements}

The Triage point-of-care immunoassay was used to determine pNGAL and UNGAL concentrations. The detection range is $46-1,300 \mathrm{ng} / \mathrm{ml}$ for the pNGAL assay and 2.6-4,100 $\mathrm{ng} /$ $\mathrm{ml}$ for the uNGAL assay. Urine samples that had concentrations exceeding the upper limit of the assay were diluted with an equal volume of $0.2 \mathrm{M} \mathrm{BES}$ buffer before application to the cartridge. The pNGAL assay on this platform has been shown to correlate strongly with other methods of pNGAL detection [24].

\section{CyC Measurements}

The Triage point-of-care immunoassay was used to measure $\mathrm{CyC}$ in both plasma and urine on a research-based cartridge. Plasma samples were measured and calibrated with a CyC-spiked plasma pool. The urine assay was calibrated with a urine pool diluted 1:1 with a buffer $(0.2 \mathrm{M} \mathrm{BES})$, which was necessary to dilute the urine samples for appropriate detection. The performance of the plasma CyC assay was validated against the commercially available Dade method. 


\section{Data Analysis}

Patients were classified into three groups: no AKI, transient AKI and sustained AKI. Demographics and clinical outcomes were summarized and compared among groups. For continuous variables, medians and interquartile ranges (IQR) were reported and the Kruskal-Wallis test was used to test for group differences. For categorical variables, frequencies and proportions were reported and Fisher's exact test was used to test for group differences.

Considering the highly skewed values in some biomarker measurements, medians and IQRs were presented for biomarker levels measured at each time point to show the temporal trend of biomarker levels over time. At each time point, the medians were compared between groups (no AKI, transient AKI and sustained AKI) for all patients and between RIFLE categories for sustained AKI patients using the rank transformation approach. Tukey-Kramer multiple comparison adjustment was utilized to adjust for the three pairs of comparisons at each time point, and adjusted $\mathrm{p}$ values were reported where multiple comparisons were involved.

To compare the discrimination of sustained AKI versus no AKI and sustained AKI versus transient AKI using the four biomarkers on admission to the ICU, receiver-operating characteristic (ROC) curves were generated and the areas under the curves (AUC) were compared to select the best-performing biomarker. The performance of the combination of uNGAL with other parameters was also investigated to show if there was any improvement in the discrimination of AKI patients.

Multivariable logistic regression analyses were conducted to assess predictors of sustained AKI and the performance of the predictive models while combining biomarkers with clinical factors. The clinical factors considered included MDRD eGFR on admission, diagnosis of sepsis and the following laboratory parameters determined on admission: $\mathrm{HCO}_{3}{ }^{-}$, BUN and WBC. A parsimonious clinical model was first determined based on stepwise backward elimination. Each of the four biomarkers was added individually to the parsimonious clinical model and the performance of the predictive models was evaluated using Akaike Information Criterion (AIC), the Hosmer-Lemeshow test of goodness of fit, AUC, net reclassification improvement (NRI) and integrated discrimination improvement (IDI).

Statistical analysis was performed using SAS version 9.2 (SAS Institute, Cary, N.C., USA), and R package Hmisc (http://CRAN.R-project.org/package = Hmisc) was used to calculate NRI and IDI. A significance level of 0.05 was used for all analyses.

\section{Results}

\section{Patients}

Seven hundred patients were initially considered to be enrolled in the study. After adopting the exclusion criteria, 98 patients were excluded. Another 92 patients were excluded because of established AKI before or on ICU admission, leaving 510 patients in the analysis. Baseline and clinical characteristics of the patients are presented in table 1. Patients with sustained AKI had a significantly higher baseline SCr, with correspondingly lower eGFR by the MDRD eGFR, lower serum $\mathrm{HCO}_{3}{ }^{-}$levels, higher BUN, higher pNGAL, uNGAL and pCyC values on admission, higher APACHE II and SOFA scores, lower 24-hour urine production, higher 24hour cumulative fluid balance and significantly increased ICU and hospital mortality.

\section{Biomarker Performance for Differentiation between No, Transient and Sustained AKI}

The temporal trends of each biomarker and SCr for patients grouped into no, transient and sustained AKI are shown in figure 1 . All four biomarkers as well as SCr showed significant differentiation between sustained AKI and no AKI at all time points (all $\mathrm{p} \leq 0.0002$ ) except for $\mathrm{uCyC}$ at the time of ICU admission ( $\mathrm{p}=0.06$; table 2). 
Table 1. Baseline characteristics and clinical outcome of the patients

\begin{tabular}{|c|c|c|c|c|}
\hline & $\begin{array}{l}\text { No AKI } \\
(\mathrm{n}=444)\end{array}$ & $\begin{array}{l}\text { Transient AKI } \\
(\mathrm{n}=19)\end{array}$ & $\begin{array}{l}\text { Sustained AKI } \\
(\mathrm{n}=47)\end{array}$ & $\mathrm{p}$ value \\
\hline Age, years & $57(43-68)$ & $60(45-69)$ & $62(54-73)$ & 0.07 \\
\hline Male, n (\%) & $250(56)$ & $16(84)$ & $30(64)$ & 0.03 \\
\hline $\mathrm{BMI}, \mathrm{kg} / \mathrm{m}^{2}$ & $24.6(22.7-27.5)$ & $24.1(21.9-27.1)$ & $26.0(23.9-27.8)$ & 0.07 \\
\hline Baseline SCr, mg/dl & $0.74(0.62-0.90)$ & $0.74(0.68-0.86)$ & $0.90(0.68-1.02)$ & 0.0007 \\
\hline MDRD eGFR, $\mathrm{ml} / \mathrm{min}$ & $100.0(86.4-100.0)$ & $100.0(92.4-100.0)$ & $70.4(52.2-93.6)$ & $<0.0001$ \\
\hline $\mathrm{SCr}, \mathrm{mg} / \mathrm{dl}$ & $0.75(0.62-0.89)$ & $0.83(0.72-0.98)$ & $1.01(0.74-1.20)$ & $<0.0001$ \\
\hline $\mathrm{HCO}^{-}, \mathrm{mmol} / \mathrm{l}$ & $22.0(20.1-24.1)$ & $21.3(19.9-23.8)$ & $19.7(17.0-21.8)$ & 0.0001 \\
\hline BUN, mmol/l & $5.4(4.2-7.0)$ & $6.0(4.2-8.5)$ & $7.2(4.9-9.4)$ & 0.006 \\
\hline WBC, $\times 10^{9} / 1$ & $11.2(8.4-14.9)$ & $9.0(5.5-13.4)$ & $10.6(6.0-12.8)$ & 0.02 \\
\hline pNGAL, ng/ml & $148.8(83.2-223.0)$ & $243.4(154.4-294.7)$ & $286.3(197.8-412.0)$ & $<0.0001$ \\
\hline uNGAL, ng/ml & $73.1(34.5-190.8)$ & $89.8(52.5-276.6)$ & $389.8(106.3-1833.2)$ & $<0.0001$ \\
\hline pCyC; mg/l & $0.9(0.8-1.2)$ & $1.1(1.0-1.3)$ & $1.3(1.0-1.8)$ & $<0.0001$ \\
\hline $\mathrm{uCyC}, \mathrm{mg} / \mathrm{l}$ & $2.6(2.6-2.7)$ & $2.6(2.6-3.5)$ & $2.6(2.6-3.9)$ & 0.05 \\
\hline APACHE II-24 score & $16(13-22)$ & $22(15-26)$ & $23(17-28)$ & $<0.0001$ \\
\hline SOFA-24 score & $4(2-6)$ & $7.5(6.0-10.0)$ & $9(7-12)$ & $<0.0001$ \\
\hline $\mathrm{UP}-24, \mathrm{ml} / \mathrm{kg} / \mathrm{h}$ & $1.1(0.8-1.7)$ & $0.9(0.6-1.3)$ & $0.8(0.5-1.3)$ & 0.004 \\
\hline $\mathrm{FB}-24, \mathrm{ml}$ & $1,949(693-3,815)$ & $4,714(1,792-5,334)$ & $4,241(1,774-6,948)$ & $<0.0001$ \\
\hline RRT, n (\%) & $0(0)$ & $0(0)$ & $7(15)$ & $<0.0001$ \\
\hline ICU length of stay, days & $4(2-8)$ & $6(4-17)$ & $10(4-17)$ & $<0.0001$ \\
\hline ICU mortality, n (\%) & $44(10)$ & $2(11)$ & $14(30)$ & 0.001 \\
\hline Hospital mortality, n (\%) & $61(14)$ & $5(26)$ & $18(38)$ & 0.0001 \\
\hline \multicolumn{5}{|l|}{ Diagnostic group, n (\%) } \\
\hline Postoperative & $163(37)$ & $2(11)$ & $8(17)$ & - \\
\hline Medical & $82(18)$ & $2(11)$ & $7(15)$ & - \\
\hline Neurological & $92(21)$ & $3(16)$ & $4(9)$ & - \\
\hline Neurotrauma & $27(6)$ & $2(11)$ & $1(2)$ & - \\
\hline Multitrauma & $30(7)$ & $4(21)$ & $4(9)$ & - \\
\hline LTx & $19(4)$ & $1(5)$ & $7(15)$ & - \\
\hline Sepsis & $12(3)$ & $1(5)$ & $5(11)$ & - \\
\hline $\mathrm{CPR}$ & $9(2)$ & $1(5)$ & $4(9)$ & - \\
\hline Hemorrhagic shock & $9(2)$ & $3(16)$ & $1(2)$ & - \\
\hline MOF & $1(0)$ & $0(0)$ & $6(13)$ & - \\
\hline
\end{tabular}

$\mathrm{BMI}=$ Body mass index; APACHE II-24 = APACHE II score during the first $24 \mathrm{~h}$; SOFA-24 = SOFA score during the first $24 \mathrm{~h}$; UP-24 = urine production during the first $24 \mathrm{~h} ; \mathrm{FB}-24=$ fluid balance during the first $24 \mathrm{~h}$; RRT = renal replacement therapy; LTx = liver transplantation; CPR = cardiopulmonary resuscitation; $\mathrm{MOF}=$ multiorgan failure. Medians $(\mathrm{IQR})$ were reported for continuous variables and frequencies (proportions) for categorical variables.

The timing of first significant differentiation between sustained AKI and transient AKI differed substantially by biomarker. uNGAL was the only biomarker to show significant differentiation between sustained and transient $\mathrm{AKI}$ on admission and at all time points thereafter (all $\mathrm{p} \leq 0.02)$; $\mathrm{uCyC}$ significantly differed at $\mathrm{T}=4(\mathrm{p}=0.03)$ and $\mathrm{T}=8 \mathrm{~h}(\mathrm{p}=0.04)$; $\mathrm{pCyC}$ first showed significant differences at $24 \mathrm{~h}(\mathrm{p}=0.005)$; pNGAL did not differ until $36 \mathrm{~h}(\mathrm{p}=0.0002$; table 2$)$.

Differentiation between transient and no AKI was more variable, with $\mathrm{pCyC}$ differing only at $4 \mathrm{~h}(\mathrm{p}=0.02)$; pNGAL differentiating only at 4 and $8 \mathrm{~h}(\mathrm{p} \leq 0.009)$; SCr differing at 24,48 and $72 \mathrm{~h}$ ( $\mathrm{p} \leq 0.0008$ ), which is as expected by definition, and UNGAL and uCyC unable to distinguish no AKI from transient AKI at any time point on or after ICU admission (table 2). 


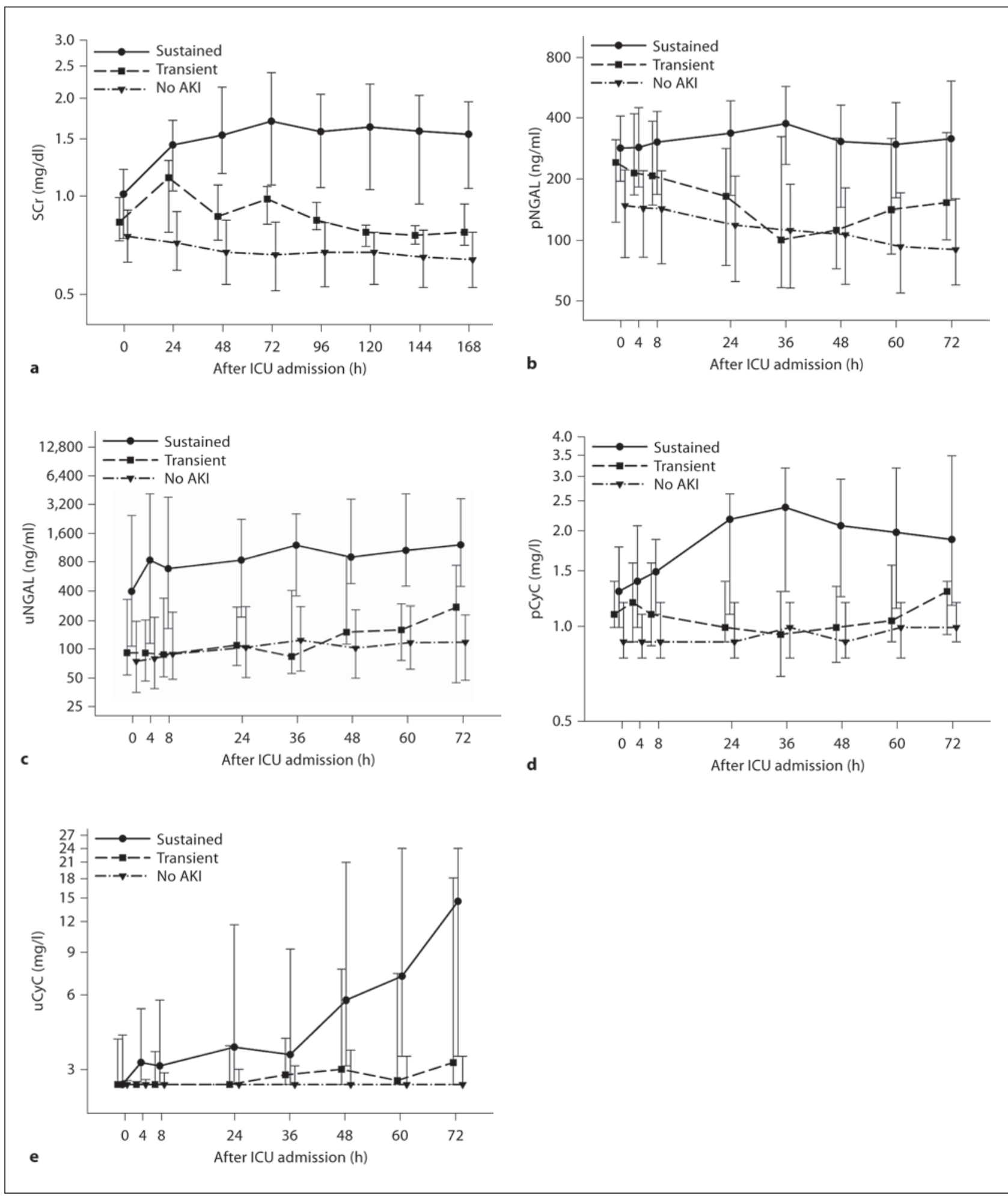

Fig. 1. Changes in the individual biomarker concentrations over time after ICU admission summarized by sustained, transient and no AKI for SCr (a) and the four biomarkers pNGAL (b), uNGAL (c), pCyC (d) and $\mathrm{uCyC}(\mathbf{e})$. Medians and IQR presented on a $\log _{10}$ scale. 
Table 2. Biomarker performance for distinguishing different disease states

\begin{tabular}{|c|c|c|c|c|c|c|c|}
\hline \multirow[t]{2}{*}{ Biomarker } & \multirow{2}{*}{$\begin{array}{l}\text { Time since } \\
\text { admission } \\
\mathrm{h}\end{array}$} & \multicolumn{3}{|l|}{ AKI } & \multicolumn{3}{|c|}{ AKI RIFLE categories } \\
\hline & & $\begin{array}{l}\text { sustained } \\
\text { vs. no AKI }\end{array}$ & $\begin{array}{l}\text { sustained } \\
\text { vs. transient }\end{array}$ & $\begin{array}{l}\text { transient } \\
\text { vs. no AKI }\end{array}$ & $\begin{array}{l}\text { failure } \\
\text { vs. injury }\end{array}$ & $\begin{array}{l}\text { failure } \\
\text { vs. risk }\end{array}$ & $\begin{array}{l}\text { injury } \\
\text { vs. risk }\end{array}$ \\
\hline \multirow[t]{8}{*}{ pNGAL } & 0 & $<0.0001$ & - & - & - & - & - \\
\hline & 4 & $<0.0001$ & - & 0.001 & - & - & - \\
\hline & 8 & $<0.0001$ & - & 0.009 & - & 0.01 & - \\
\hline & 24 & $<0.0001$ & - & - & - & 0.02 & - \\
\hline & 36 & $<0.0001$ & 0.0002 & - & - & 0.03 & - \\
\hline & 48 & $<0.0001$ & 0.02 & - & - & 0.03 & - \\
\hline & 60 & $<0.0001$ & - & - & - & - & - \\
\hline & 72 & $<0.0001$ & - & - & - & 0.03 & - \\
\hline \multirow[t]{8}{*}{ uNGAL } & 0 & $<0.0001$ & 0.02 & - & - & - & - \\
\hline & 4 & $<0.0001$ & 0.01 & - & - & 0.04 & - \\
\hline & 8 & $<0.0001$ & 0.002 & - & - & - & - \\
\hline & 24 & $<0.0001$ & 0.004 & - & - & 0.02 & 0.03 \\
\hline & 36 & $<0.0001$ & $<0.0001$ & - & - & - & - \\
\hline & 48 & $<0.0001$ & 0.002 & - & - & - & - \\
\hline & 60 & $<0.0001$ & 0.0008 & - & - & - & - \\
\hline & 72 & $<0.0001$ & 0.01 & - & - & 0.049 & - \\
\hline \multirow[t]{8}{*}{ pCyC } & 0 & $<0.0001$ & - & - & - & - & - \\
\hline & 4 & $<0.0001$ & - & 0.02 & - & - & - \\
\hline & 8 & $<0.0001$ & - & - & - & - & - \\
\hline & 24 & $<0.0001$ & 0.005 & - & - & - & - \\
\hline & 36 & $<0.0001$ & $<0.0001$ & - & - & - & - \\
\hline & 48 & $<0.0001$ & 0.0001 & - & - & - & - \\
\hline & 60 & $<0.0001$ & 0.01 & - & - & - & - \\
\hline & 72 & $<0.0001$ & - & - & - & - & - \\
\hline \multirow[t]{8}{*}{ uCyC } & 0 & - & - & - & - & - & - \\
\hline & 4 & $<0.0001$ & 0.03 & - & - & - & - \\
\hline & 8 & $<0.0001$ & 0.04 & - & - & - & - \\
\hline & 24 & $<0.0001$ & - & - & - & - & - \\
\hline & 36 & 0.0002 & - & - & - & - & - \\
\hline & 48 & $<0.0001$ & - & - & - & - & - \\
\hline & 60 & $<0.0001$ & - & - & - & - & - \\
\hline & 72 & $<0.0001$ & - & - & - & - & - \\
\hline
\end{tabular}

Biomarker Performance for Differentiation of AKI Severity

Patients with sustained AKI were divided into three groups based on their RIFLE categories $(\mathrm{R}=$ risk 1.5 - to 2.0-fold increase in baseline SCr; $\mathrm{I}=$ injury 2.0-3.0 times, and $\mathrm{F}=$ failure $>3$ times; $\mathrm{n}=47$ ). The temporal trends for each biomarker and $\mathrm{SCr}$ are shown in figure 2. Neither pCyC nor uCyC showed significant differentiation among the RIFLE categories at any time. pNGAL did not significantly differentiate between $\mathrm{F}$ and I or between I and $\mathrm{R}$, but started to show differentiation between F and $\mathrm{R} 8 \mathrm{~h}$ after ICU admission $(\mathrm{p}=0.01)$. The differentiation of RIFLE categories using uNGAL was similar to pNGAL. uNGAL only showed significant differentiation between $\mathrm{F}$ and $\mathrm{R}$ at $4(\mathrm{p}=0.04), 24(\mathrm{p}=0.02)$ and $72 \mathrm{~h}$ $(\mathrm{p}=0.049)$, and between $\mathrm{I}$ and $\mathrm{R}$ at $24 \mathrm{~h}(\mathrm{p}=0.03)$. On and $24 \mathrm{~h}$ after ICU admission, SCr did not show significant discriminative power among RIFLE categories, and only enabled to differentiate F versus $\mathrm{R}$ at $48 \mathrm{~h}(\mathrm{p}=0.0004)$ and F versus I at $72 \mathrm{~h}(\mathrm{p}=0.03$; table 2$)$. 


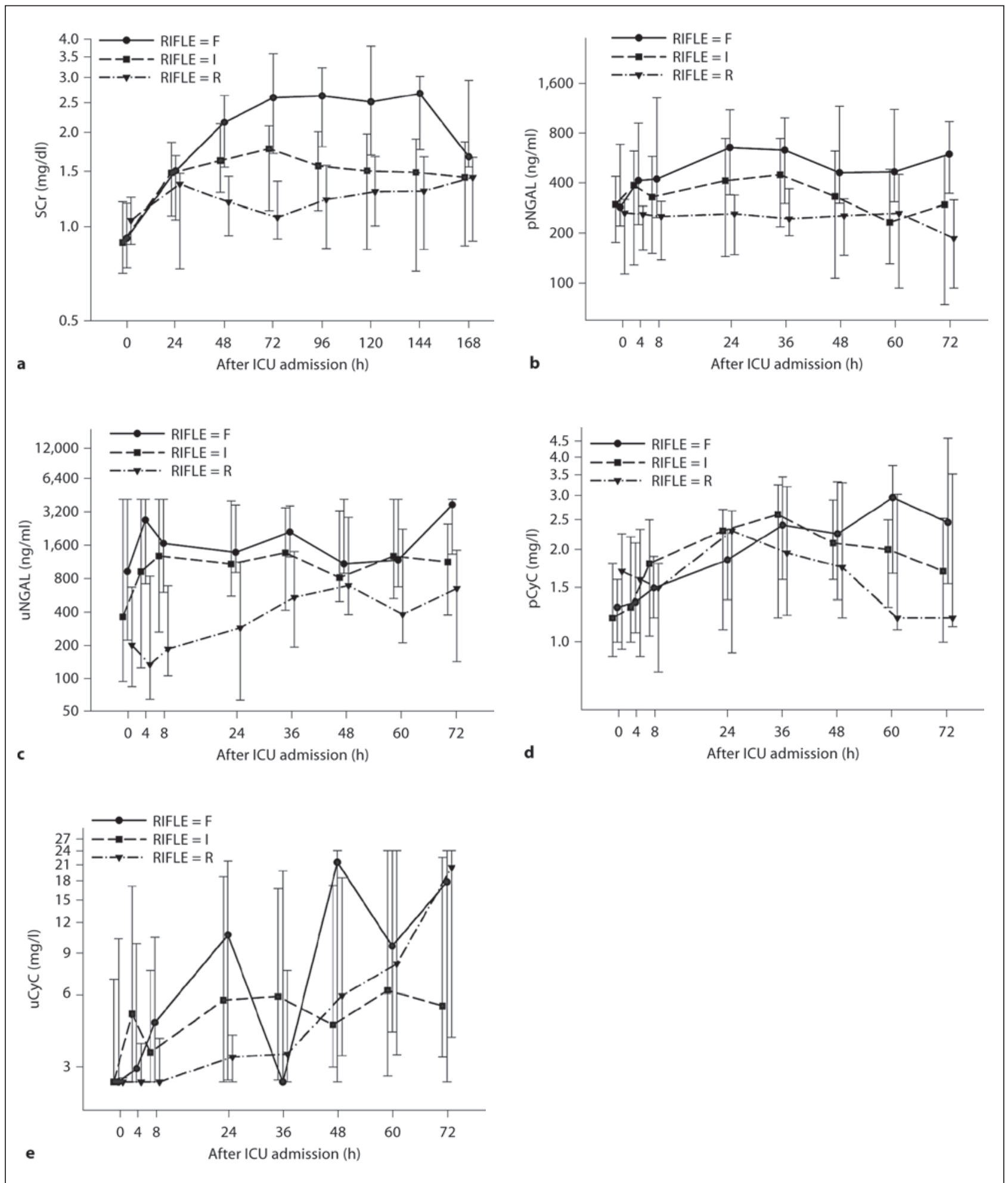

Fig. 2. Changes in the individual biomarker concentrations over time after ICU admission summarized by RIFLE stages risk (R), injury (I) and failure (F) for SCr (a) and the four biomarkers pNGAL (b), uNGAL (c), $\mathrm{pCyC}(\mathbf{d})$ and $\mathrm{uCyC}(\mathbf{e})$. Medians and IQR presented on a $\log _{10}$ scale. 


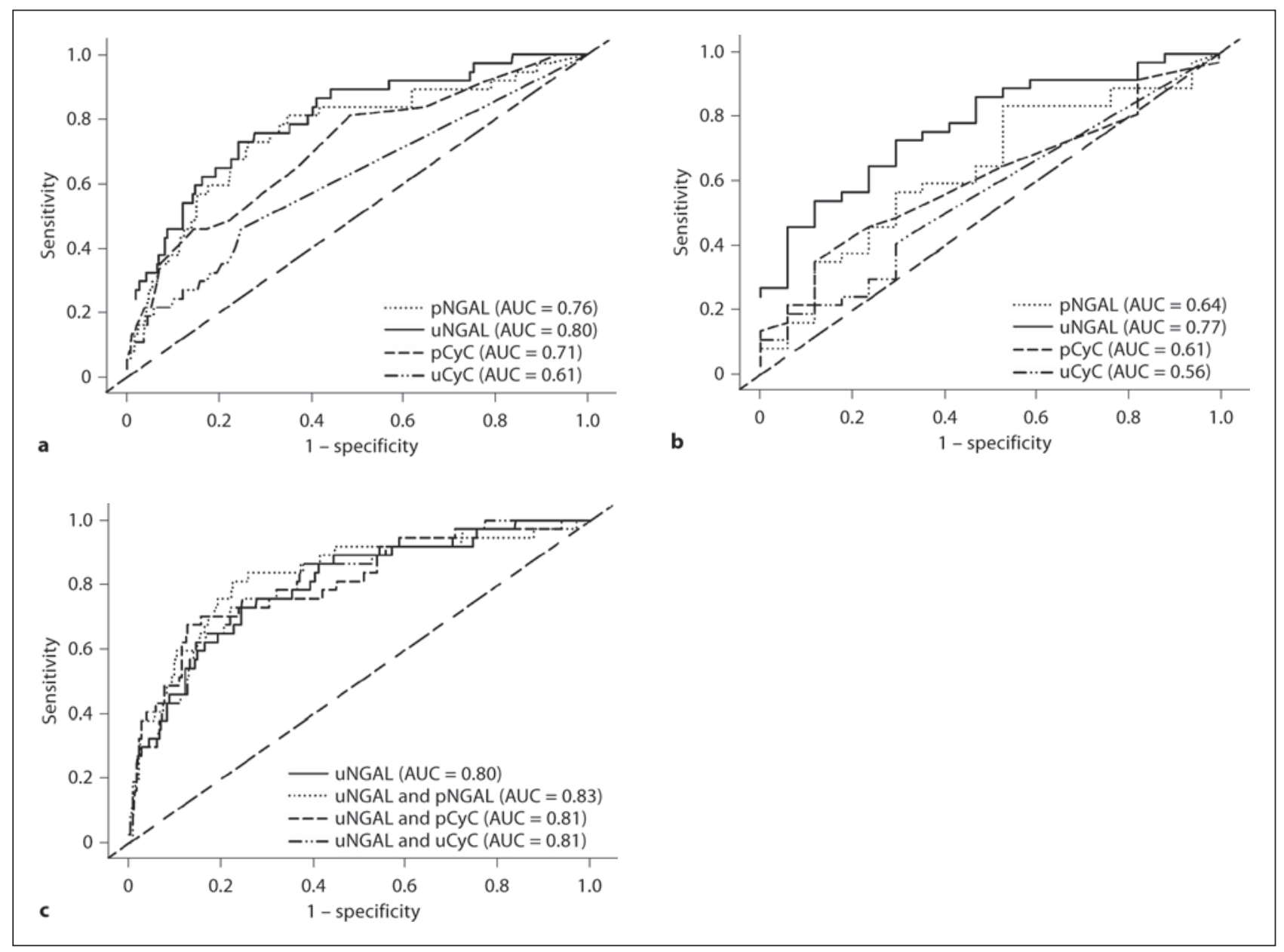

Fig. 3. a ROC curves and corresponding AUCs for the prediction of sustained AKI ( $\mathrm{n}=37$ ) versus no AKI $(\mathrm{n}=375)$ on ICU admission for pNGAL, uNGAL, pCyC and uCyC. b ROC curves and corresponding AUCs for the prediction of sustained $(n=37)$ versus transient AKI $(n=17)$ on ICU admission for pNGAL, uNGAL, pCyC and uCyC. c ROC curves and corresponding AUCs for the prediction of sustained $(\mathrm{n}=37)$ versus no AKI $(\mathrm{n}=375)$ on ICU admission for uNGAL in combination with SCr, pNGAL, pCyC and uCyC.

Performance of Biomarkers Measured on Admission in the Prediction of Sustained AKI

To compare the performance of each individual biomarker in the prediction of AKI and to investigate the performance of combinations of biomarkers, subjects who had missing values in one or more biomarkers at the time of admission were excluded for ROC curve analysis.

Four hundred and seventeen patients were included to investigate the discrimination of sustained AKI (RIFLE $\mathrm{R}$ and above for $>24 \mathrm{~h}, \mathrm{n}=37$ ) versus no AKI $(\mathrm{n}=380)$ using different biomarkers. All studied biomarkers (pNGAL, uNGAL, pCyC and $\mathrm{uCyC}$ ) were significant predictors of sustained AKI $(\mathrm{p}<0.005)$. uNGAL showed the best discrimination of sustained AKI versus no AKI with an AUC of 0.80 (95\% confidence interval, $\mathrm{CI}=0.72-0.88$ ) compared to pNGAL ( $\mathrm{AUC}=0.76,95 \% \mathrm{CI}=0.67-0.85)$, $\mathrm{pCyC}(\mathrm{AUC}=0.71,95 \% \mathrm{CI}=0.62-0.80)$ and $\mathrm{uCyC}(\mathrm{AUC}=0.61,95 \% \mathrm{CI}=0.52-0.70$; fig. $3 \mathrm{a})$. There was no significant difference in AUC between uNGAL and pNGAL, or between uNGAL and pCyC, but AUC were significantly higher for uNGAL than $\mathrm{uCyC}(\mathrm{p}<0.0001)$. Regarding the discrimination of sustained AKI 
Table 3. Comparison of the performance of the predictive models after adding the biomarkers

\begin{tabular}{|c|c|c|c|c|c|c|}
\hline Model & $\begin{array}{l}\text { Biomarker } \\
\text { ( } \mathrm{p} \text { value) }\end{array}$ & AIC & $\begin{array}{l}\text { H-L test } \\
\text { (p value) }\end{array}$ & AUC (95\% CI) & $\begin{array}{l}\text { NRI } \\
\text { (p value) }\end{array}$ & $\begin{array}{l}\text { IDI } \\
\text { (p value) }\end{array}$ \\
\hline Clinical model & - & 197.2 & 0.01 & $0.79(0.70-0.89)$ & - & - \\
\hline Clinical + uNGAL & 0.0008 & 188.7 & 0.21 & $0.82(0.73-0.91)$ & $0.38(0.04)$ & $0.06(0.04)$ \\
\hline Clinical + pNGAL & 0.052 & 195.5 & 0.01 & $0.81(0.72-0.90)^{*}$ & $0.42(0.02)$ & $0.02(0.28)$ \\
\hline Clinical + uCyC & 0.76 & 199.1 & 0.01 & $0.79(0.70-0.89)$ & $-0.25(0.17)$ & $0.0004(0.88)$ \\
\hline Clinical + pCyC & 0.006 & 191.3 & 0.02 & $0.81(0.72-0.90)$ & $0.08(0.67)$ & $0.04(0.09)$ \\
\hline
\end{tabular}

$\mathrm{H}-\mathrm{L}=$ Hosmer-Lemeshow. ${ }^{*} \mathrm{p}<0.05$ for improvement in AUC vs. clinical model alone.

$(\mathrm{n}=37)$ versus transient AKI $(\mathrm{n}=17)$, uNGAL was the only biomarker which showed significance with an AUC of 0.77 (95\% CI = 0.64-0.90; fig. 3b).

Biomarker Combinations for the Prediction of Sustained AKI Measured on Admission

Combining uNGAL with one of the other three biomarkers did improve the AUCs for the discrimination of sustained versus no AKI; however, the improvements were not statistically significant. The best combination was UNGAL and pNGAL, improving the AUC to 0.83 $(95 \% \mathrm{CI}=0.75-0.91)$ from 0.80 with uNGAL alone (fig. $3 \mathrm{c}$ ). There is no improvement in combining biomarkers for the discrimination between sustained and transient AKI.

\section{Multivariable Logistic Regression Analysis for the Prediction of Sustained AKI}

To have a fair comparison between the biomarkers, patients with missing values in any of the five clinical variables (MDRD eGFR, $\mathrm{HCO}_{3}{ }^{-}, \mathrm{BUN}, \mathrm{WBC}$ and sepsis) and the four biomarkers at the time of ICU admission were excluded from the multivariable logistic regression analysis. This resulted in a total of 389 patients (33 sustained AKI vs. 356 others). After a stepwise backward elimination, $\mathrm{MDRD}$ eGFR, $\mathrm{HCO}_{3}{ }^{-}$and diagnosis of sepsis remained in the parsimonious clinical model as independent predictors of sustained AKI ( $p=0.002$, $p=0.045$ and $p=0.0001$, respectively). The additional contribution of biomarkers to the prediction of sustained AKI was evaluated by adding each biomarker individually to the parsimonious clinical model (table 3). uNGAL and pCyC proved to be significant predictors of sustained AKI even with the existence of the three clinical factors. pNGAL was marginally significant $(\mathrm{p}=0.052)$ and $\mathrm{uCyC}$ was not significant $(\mathrm{p}=0.76)$ after they were added to the parsimonious clinical model. The model with uNGAL showed the best goodness of fit based on the comparison of AIC (decrease $>7$ in the magnitude of AIC from the clinical model) and the Hosmer-Lemeshow test $(\mathrm{p}=0.21)$. There was minor improvement in AUC (0.0180.024 ) when combining a biomarker with the parsimonious clinical model, but the improvement was not statistically significant except for pNGAL ( $\mathrm{p}=0.03)$. Additional evaluation of the performance of the combined models was conducted by calculating NRI and IDI for each combined model versus the parsimonious clinical model. Since no standard scale is available for the choice of classification risk levels, NRI was calculated based on the increase or decrease of predicted individual probability of developing sustained AKI. Significance was shown in NRI for models containing uNGAL (NRI $=0.38, \mathrm{p}=0.04)$ or $\mathrm{pNGAL}(\mathrm{NRI}=0.42$, $\mathrm{p}=0.02$ ). Another index for the evaluation of the performance of a predictive model, IDI, showed significant improvement for the model containing uNGAL (IDI $=0.06, \mathrm{p}=0.04$ ). Considering the components of IDI, the combined model with uNGAL improved classification specificity significantly $(\mathrm{p}=0.04)$. 


\section{Discussion}

This study is the first to our knowledge to demonstrate the utility of uNGAL as a biomarker that could be used upon admission to the ICU to distinguish critically ill adults who are likely to develop sustained versus transient AKI from those at minimal risk of AKI. Such an early clinical determination may allow for the alteration in clinical care toward prevention and early treatment of AKI, prior to the rise in SCr typically used to diagnose AKI. However, this study also suggests that NGAL and $\mathrm{CyC}$, either alone or in combination, are not able to accurately or persistently discriminate AKI RIFLE severity among critically ill patients with developing AKI. Furthermore, although based upon a small event sample size the results suggest modest additional value of the markers when added to a model with clinical variables. These negative findings are also important, as they suggest important limitations of these biomarkers in this critical-care setting.

The cellular and molecular aspects of ischemic and septic AKI are currently being further elucidated. Major progress has been made in the knowledge of the underlying pathophysiological mechanisms and possible targets for therapeutic interventions. However, the translation of these findings to clinical patient-related practice has been disappointing. Different pathophysiological stages of AKI have been identified, including a prerenal, initiation, extension and maintenance phase. Therapy must be considered in the context of these different stages, because applying them in the maintenance phase has proven to be unsuccessful. Therefore, early recognition and discrimination of the first stages of AKI, not yet reflected by functional impairment, are very important $[5,25]$. To our knowledge, the data presented show for the first time that uNGAL is able to provide information on the discrimination between sustained and transient AKI in the early clinical course of AKI development, upon admission to the ICU. Such information may prove useful in the early initiation of several emerging treatment modalities, such as innate immune system modulators, growth factors, regulatory $\mathrm{T}$ cells, cytoprotective agents such as activated protein C, statins and erythropoietin. In addition, there are issues we would potentially address differently in current practice in patients destined for sustained AKI if we could discriminate them from patients with self-limiting renal injury. For example, we would attempt a more intense regime of 'golden hour resuscitation' with close hemodynamic monitoring of macro- and microcirculation, we would avoid nephrotoxic agents and most importantly we would attempt more timely initiation of renal replacement therapy to at least control cumulative fluid balances and toxic uremic conditions that are clearly correlated with survival and ventilator-free days [26-31].

Several studies report promising results for NGAL (AUC ranging from 0.71 to 0.92 ) [13-16, 32] and CyC (AUCs ranging from 0.50 to 0.78) [18, 19, 33-35] for AKI prediction in adult critically ill patients. However, the AUC results are not very consistent and highly depended on the selected patient cohort (e.g. general ICU patients vs. cardiopulmonary bypass surgery patients vs. septic patients), and on the inclusion or exclusion of preexisting CKD or preexisting AKI. Therefore, newer trends in biomarker research have been directed towards the use of biomarker models that include clinical parameters to improve the test accuracy for early detection of AKI in well-defined cohorts [36-38].

The current study excluded patients with known CKD or AKI, improving the homogeneity of our cohort and allowing for an assessment of incident AKI only. It also describes the individual and combined performance of four different biomarkers (pNGAL, uNGAL, pCyC and $\mathrm{uCyC}$ ) allowing for their direct comparison. In this analysis, the prediction of sustained AKI was not significantly different when considering the ROC AUC between pNGAL, uNGAL, $\mathrm{pCyC}$ and $\mathrm{uCyC}$. This may be partly explained by the reduction in sample size using patients with developing AKI only. Adding the individual biomarkers to a model with clinical characteristics, the improvement in the combined model with uNGAL, pNGAL and pCyC was com- 
parable, and therefore any potential superiority of a biomarker could not be demonstrated, except for $\mathrm{uCyC}$, which was not a significant predictor in the multivariable model.

Several issues have to be considered concerning potential limitations of this current work.

(1) Defining a patient's baseline SCr is a problem in emergency medicine. Not uncommonly, these variables are not available due to the absence of the patient's medical history data in the hospital charts. Using the first available SCr value, such as we did, might therefore be a reason for some underestimation of AKI severity and might contribute to biomarker test nonspecificity. However, the procedures we employed in this study are consistent with the clinical determination of AKI. Furthermore, our results have to be considered in a researchbased setting and may not reflect entirely common clinical practice. For the purpose of the analysis, this cohort was restricted to patients with developing AKI only, and thus patients having reached an increase of $\geq 50 \%$ in SCr before or on admission were excluded. This is only possible with the availability of the the patient's previous SCr baseline values. It is very common, however, that in clinical practice the information on a patient's true baseline SCr value is not immediately available and thus the clinical applicability of the differentiation between transient and sustained AKI remains to be proven as having additional value.

(2) We have to take into account that new biomarkers such as NGAL (injury marker) and $\mathrm{CyC}$ (functional marker) are tested against a gold standard that has huge imperfections on its own. Therefore, we should keep in mind that the lack of sensitivity and specificity of a biomarker in the present approach may actually reflect limited use of SCr [6].

(3) Urinary values of biomarkers are not corrected for urinary creatinine concentrations; however, this is defendable since there is evidence that it is not likely to have a considerable impact on overall test performance [39].

(4) In this study, exclusion of all patients with CKD rendered the results possibly less applicable to the general adult intensive-care population where we observe increasing numbers of patients entering the ICU with CKD.

(5) pNGAL and uNGAL were the only inducible damage markers used in this panel. The additional contribution of $\mathrm{uCyC}$ in this model was disappointing.

Our findings are in contrast with the conclusions of the work of Koyner et al. [34] and several others stating that CyC may be an improved estimator of GFR particularly in patients with very subtle changes in kidney function and injury $[18,19,38,40]$. Recent data demonstrate that in the absence of CKD, serum CyC and $\mathrm{uCyC}$ may be associated with inflammatory biomarkers in an elderly ambulatory population and sepsis in general ICU patients [41, 42]. A possible hypothesis is that $\mathrm{CyC}$ itself may be a regulator of inflammation, which might affect particularly adult critically ill patients with known proinflammatory state. Accordingly, subgroups of this population frequently receive corticosteroid treatment, e.g. sepsis and liver transplant patients, and patients suffer from thyroid dysfunction syndromes. Using other particular damage markers such as KIM-1 (kidney injury molecule-1) [43], interleukin-18 [44], netrin-1 [45, 46] or liver fatty acid binding proteins [47] might potentially have a significant additional predictive value to the biomarker panel model, which should be a subject of exploration in the near future.

In conclusion, we showed that uNGAL accurately discriminates adult patients likely to develop sustained AKI from transient AKI at the time of ICU admission. In addition, NGAL and $\mathrm{CyC}$, either alone or in combination, were not able to accurately or persistently discriminate AKI RIFLE severity among critically ill patients with developing AKI. However, adding uNGAL, pNGAL and pCyC to a model with clinical characteristics slightly improved the discrimination of the predictive model, and especially adding UNGAL to the clinical model significantly improved the performance of the prediction in terms of both NRI and IDI. 


\section{Acknowledgments}

We thank the clinical and laboratory staff of the Erasmus University Medical Center, Rotterdam, The Netherlands. We thank Wil Mol, our nurse coordinator, for her contribution in patient inclusion and study logistics, as well as the patients and their families for their participation. We thank Gillian Parker and Brian Noland from Biosite Inc. (San Diego, Calif., USA) for the measurements of NGAL and CyC levels.

\section{Disclosure Statement}

Biosite Inc. provided biomarker measurements. They had no role in study design and conduct, data analysis and reporting. Besides biomarker assessments, no additional funding was provided.

\section{References}

1 Liangos O, Wald R, O'Bell JW, et al: Epidemiology and outcomes of acute renal failure in hospitalized patients: a national survey. Clin J Am Soc Nephrol 2006;1:43-51.

2 Metnitz PG, Krenn CG, Steltzer H, et al: Effect of acute renal failure requiring renal replacement therapy on outcome in critically ill patients. Crit Care Med 2002;30:2051-2058.

3 Uchino S, Kellum JA, Bellomo R, et al: Acute renal failure in critically ill patients: a multinational, multicenter study. JAMA 2005;294:813-818.

4 Uchino S, Bellomo R, Bagshaw SM, et al: Transient azotaemia is associated with a high risk of death in hospitalized patients. Nephrol Dial Transplant 2010;25:1833-1839.

5 Molitoris BA: Transitioning to therapy in ischemic acute renal failure. J Am Soc Nephrol 2003;14: $265-267$.

6 Waikar SS, Betensky RA, Bonventre JV: Creatinine as the gold standard for kidney injury biomarker studies? Nephrol Dial Transplant 2009;24:3263-3265.

7 Flo TH, Smith KD, Sato S, et al: Lipocalin 2 mediates an innate immune response to bacterial infection by sequestrating iron. Nature 2004;432:917-921.

8 Mishra J, Mori K, Ma Q, et al: Amelioration of ischemic acute renal injury by neutrophil gelatinaseassociated lipocalin. J Am Soc Nephrol 2004;15:3073-3082.

9 Schmidt-Ott KM, Mori K, Kalandadze A, et al: Neutrophil gelatinase-associated lipocalin-mediated iron traffic in kidney epithelia. Curr Opin Nephrol Hypertens 2006;15:442-449.

10 Schmidt-Ott KM, Mori K, Li JY, et al: Dual action of neutrophil gelatinase-associated lipocalin. J Am Soc Nephrol 2007;18:407-413.

11 Dharnidharka VR, Kwon C, Stevens G: Serum cystatin C is superior to serum creatinine as a marker of kidney function: a meta-analysis. Am J Kidney Dis 2002;40:221-226.

12 Nejat M, Pickering JW, Walker RJ, Endre ZH: Rapid detection of acute kidney injury by plasma cystatin C in the intensive care unit. Nephrol Dial Transplant 2010;25:3283-3289.

13 Constantin JM, Futier E, Perbet S, et al: Plasma neutrophil gelatinase-associated lipocalin is an early marker of acute kidney injury in adult critically ill patients: a prospective study. J Crit Care 2010;25: 176.e1-176.e6.

14 Cruz DN, de Cal M, Garzotto F, et al: Plasma neutrophil gelatinase-associated lipocalin is an early biomarker for acute kidney injury in an adult ICU population. Intensive Care Med 2010;36:444-451.

15 Siew ED, Ware LB, Gebretsadik T, et al: Urine neutrophil gelatinase-associated lipocalin moderately predicts acute kidney injury in critically ill adults. J Am Soc Nephrol 2009;20:1823-1832.

16 de Geus HR, Bakker J, Lesaffre EM, le Noble JL: Neutrophil gelatinase-associated lipocalin at ICU admission predicts for acute kidney injury in adult patients. Am J Respir Crit Care Med 2011;183: 907-914. 
17 Ahlstrom A, Tallgren M, Peltonen S, et al: Evolution and predictive power of serum cystatin C in acute renal failure. Clin Nephrol 2004;62:344-350.

18 Herget-Rosenthal S, Marggraf G, Hüsing J, et al: Early detection of acute renal failure by serum cystatin C. Kidney Int 2004;66:1115-1122.

19 Herget-Rosenthal S, Poppen D, Hüsing J, et al: Prognostic value of tubular proteinuria and enzymuria in nonoliguric acute tubular necrosis. Clin Chem 2004;50:552-558.

20 Villa P, Jiménez M, Soriano MC, et al: Serum cystatin C concentration as a marker of acute renal dysfunction in critically ill patients. Crit Care 2005;9:R139-R143.

21 Jansen TC, Kompanje EJ, Bakker J: Deferred proxy consent in emergency critical care research: ethically valid and practically feasible. Crit Care Med 2009;37(1 suppl):S65-S68.

22 Levey AS, Bosch JP, Lewis JB, et al: A more accurate method to estimate glomerular filtration rate from serum creatinine: a new prediction equation. Modification of Diet in Renal Disease Study Group. Ann Intern Med 1999;130:461-470.

23 Bellomo R, Ronco C, Kellum JA, et al: Acute renal failure - definition, outcome measures, animal models, fluid therapy and information technology needs: the Second International Consensus Conference of the Acute Dialysis Quality Initiative (ADQI) Group. Crit Care 2004;8:R204-R212.

24 Dent CL, Ma Q, Dastrala S, et al: Plasma neutrophil gelatinase-associated lipocalin predicts acute kidney injury, morbidity and mortality after pediatric cardiac surgery: a prospective uncontrolled cohort study. Crit Care 2007;11:R127.

25 Molitoris BA, Sutton TA: Endothelial injury and dysfunction: role in the extension phase of acute renal failure. Kidney Int 2004;66:496-499.

26 Rivers E, Nguyen B, Havstad S, et al: Early goal-directed therapy in the treatment of severe sepsis and septic shock. N Engl J Med 2001;345:1368-1377.

27 Demirkiliç U, Kuralay E, Yenicesu M, et al: Timing of replacement therapy for acute renal failure after cardiac surgery. J Card Surg 2004;19:17-20.

28 Elahi MM, Lim MY, Joseph RN, et al: Early hemofiltration improves survival in post-cardiotomy patients with acute renal failure. Eur J Cardiothorac Surg 2004;26:1027-1031.

29 Liu KD, Himmelfarb J, Paganini E, et al: Timing of initiation of dialysis in critically ill patients with acute kidney injury. Clin J Am Soc Nephrol 2006;1:915-919.

30 Payen D, de Pont AC, Sakr Y, et al: A positive fluid balance is associated with a worse outcome in patients with acute renal failure. Crit Care 2008;12:R74.

31 National Heart, Lung, and Blood Institute Acute Respiratory Distress Syndrome (ARDS) Clinical Trials Network, Wiedemann HP, Wheeler AP, et al: Comparison of two fluid-management strategies in acute lung injury. N Engl J Med 2006;354:2564-2575.

32 Nickolas TL, O’Rourke MJ, Yang J, et al: Sensitivity and specificity of a single emergency department measurement of urinary neutrophil gelatinase-associated lipocalin for diagnosing acute kidney injury. Ann Intern Med 2008;148:810-819.

33 Haase-Fielitz A, Bellomo R, Devarajan P, et al: Novel and conventional serum biomarkers predicting acute kidney injury in adult cardiac surgery - a prospective cohort study. Crit Care Med 2009;37: 553-560.

34 Koyner JL, Bennett MR, Worcester EM, et al: Urinary cystatin C as an early biomarker of acute kidney injury following adult cardiothoracic surgery. Kidney Int 2008;74:1059-1069.

35 Liangos O, Tighiouart H, Perianayagam MC, et al: Comparative analysis of urinary biomarkers for early detection of acute kidney injury following cardiopulmonary bypass. Biomarkers 2009;14:423431.

36 Nolen B, Velikokhatnaya L, Marrangoni A, et al: Serum biomarker panels for the discrimination of benign from malignant cases in patients with an adnexal mass. Gynecol Oncol 2010;117:440-445.

37 Shapiro NI, Trzeciak S, Hollander JE, et al: A prospective, multicenter derivation of a biomarker panel to assess risk of organ dysfunction, shock, and death in emergency department patients with suspected sepsis. Crit Care Med 2009;37:96-104.

38 Coca SG, Yalavarthy R, Concato J, et al: Biomarkers for the diagnosis and risk stratification of acute kidney injury: a systematic review. Kidney Int 2008;73:1008-1016.

39 Mishra J, Dent C, Tarabishi R, et al: Neutrophil gelatinase-associated lipocalin (NGAL) as a biomarker for acute renal injury after cardiac surgery. Lancet 2005;365:1231-1238.

40 Laterza OF, Price CP, Scott MG: Cystatin C: an improved estimator of glomerular filtration rate? Clin Chem 2002;48:699-707. 
41 Keller CR, Odden MC, Fried LF, et al: Kidney function and markers of inflammation in elderly persons without chronic kidney disease: the health, aging, and body composition study. Kidney Int 2007; 71:239-244.

42 Nejat M, Pickering JW, Walker RJ, et al: Urinary cystatin C is diagnostic of acute kidney injury and sepsis, and predicts mortality in the intensive care unit. Crit Care 2010;14:R85.

43 Liangos O, Perianayagam MC, Vaidya VS, et al: Urinary N-acetyl- $\beta$-D-glucosaminidase activity and kidney injury molecule-1 level are associated with adverse outcomes in acute renal failure. J Am Soc Nephrol 2007;18:904-912.

44 Parikh CR, Mishra J, Thiessen-Philbrook H, et al: Urinary IL-18 is an early predictive biomarker of acute kidney injury after cardiac surgery. Kidney Int 2006;70:199-203.

45 Brian Reeves W, Kwon O, Ramesh G: Netrin-1 and kidney injury. II. Netrin-1 is an early biomarker of acute kidney injury. Am J Physiol Renal Physiol 2008;294:F731-F738.

46 Ramesh G, Krawczeski CD, Woo JG, Wang Y, Devarajan P: Urinary netrin-1 is an early predictive biomarker of acute kidney injury after cardiac surgery. Clin J Am Soc Nephrol 2010;5:395-401.

47 Portilla D, Dent C, Sugaya T, et al: Liver fatty acid-binding protein as a biomarker of acute kidney injury after cardiac surgery. Kidney Int 2008;73:465-472. 